\title{
Altering the Cognitive-Affective Dysfunctions of Psychopathic and Externalizing Offender Subtypes with Cognitive Remediation
}

\author{
Arielle R. Baskin-Sommers, Ph.D. ${ }^{1,2,{ }^{\star}}$, John J. Curtin, Ph.D. ${ }^{2}$, and Joseph P. Newman, Ph.D. \\ 2 \\ ${ }^{1}$ Yale University, Psychology Department \\ 2University of Wisconsin-Madison, Psychology Department
}

\begin{abstract}
Cognitive remediation is a treatment approach with the potential to translate basic science into more specific, mechanism-based interventions by targeting particular cognitive skills. The present study translated understanding of two well-defined cognitive-emotion dysfunctions into novel deficit-matched interventions and evaluated whether cognitive remediation would demonstrate specific and generalizable change. Two antisocial-subtypes, individuals with psychopathy and externalizing traits, are characterized by cognitive-affective problems that predispose them to engage in significant substance abuse and criminal behavior, culminating in incarceration. Whereas individuals with psychopathy fail to consider important contextual information, individuals with externalizing traits lack the capacity to regulate affective reactions. Training designed to remedy these subtype-specific deficits led to improvement on both trained and nontrained tasks. Such findings offer promise for changing neural and behavioral patterns, even for what many consider to be the most recalcitrant treatment population, and presage a new era of translating cognitive-affective science into increasingly specific and effective interventions.
\end{abstract}

Cognitive impairments are a persistent and functionally relevant feature of most mental health problems. Such impairments span multiple domains and are more closely linked to functional outcomes than severity of clinical symptomology, making them a treatment priority (Trivedi, 2006). In the last decade, there has been strong interest in understanding the mechanisms of behavior change and developing effective treatments that capitalize on this understanding. One particularly promising and innovative treatment strategy, cognitive remediation, attempts to train individuals in cognitive skills that have been found to be deficient in various forms of psychopathology (Klingberg, 2010). Most prominently, researchers have evaluated the efficacy of cognitive remediation as a strategy for improving

\footnotetext{
Corresponding Author: Arielle Baskin-Sommers, Ph.D., Yale University, Department of Psychology, P.O. Box 208205, New Haven, CT 06520, 203-432-4500, arielle.baskin-sommers@yale.edu.

Author Contributions

All authors contributed to the study concept and design. Testing and data collection were performed by A.R. Baskin-Sommers. A.R. Baskin-Sommers performed the data analysis and interpretation under the supervision of J.P. Newman and J.J. Curtin. A.R. BaskinSommers drafted the manuscript, and J.P. Newman and J.J. Curtin provided critical revisions. All authors approved the final version of the manuscript for submission.
} 
working memory in disorders with known executive function abnormalities, such as attention deficit-hyperactivity disorder and schizophrenia (Shipstead, Redick, \& Engle, 2012; Stevenson, Whitmont, Bornholt, Livesey, \& Stevenson, 2002; Wykes, Huddy, Cellard, McGurk, \& Czobor, 2011). While the prospect of cognitive remediation has been met with high enthusiasm, its application to various populations has been limited and the crucial test of assessing the translation of specific skills to generalizable skills has not been rigorously evaluated. As highlighted in recent prominent critiques, cognitive remediation will be an abstract hope rather than a meaningful advance until these central issues are remedied (Shipstead et al., 2012). In the present study, we go a step further by, first, using progress made in specifying unique cognitive-affective processes associated with two antisocial subtypes to develop distinct cognitive remediation interventions and, second, evaluating the extent to which the interventions demonstrate specific and generalizable change in these processes.

Two antisocial subtypes, offenders with psychopathy and externalizing traits ${ }^{1}$, are associated with significantly higher rates of antisocial activity and substance abuse than other offenders, and represent particularly high-risk populations. Reflecting their high risk, these individuals account for the majority of failed treatment efforts within the penal system (Barbaree, 2005; Compton, Cottler, Jacobs, Ben-Abdallah, \& Spitznagel, 2003; Salekin, Worley, \& Grimes, 2010). Although offenders with psychopathy and externalizing traits are relatively resistant to traditional therapies, quite likely because they were not designed to address the relatively unique cognitive-affective dysfunctions associated with these serious subtypes, advances in knowledge concerning cognitive remediation highlight new treatment options for addressing their psychopathology.

Over the last several decades, discoveries in neurobiology, cognitive neuroscience, and other science disciplines have led to significant revisions to our understanding of the etiopathogenesis of antisocial behavior. While individuals with psychopathy and those with externalizing traits have similar phenotypic expressions, including violent behavior, impulsivity, and substance abuse, they are associated with distinct cognitive-affective dysfunctions (Hare, 2006; Hicks, Markon, Patrick, Krueger, \& Newman, 2004; Patterson \& Newman, 1993).

Broadly speaking, the behavior of individuals with psychopathy reflects a callous, fearless, irresponsible disposition that stems from a lack of self-monitoring and emotional depth. Some studies report blunted reactivity to aversive events and poor fear conditioning in

\footnotetext{
${ }^{1}$ Psychopathy is generally conceptualized as a diagnostic syndrome comprised of numerous individual traits, such as impulsivity, irresponsibility, shallow affect, and glibness, to varying degrees. In contrast, externalizing, by definition, is not intended to identify a specific disorder or set of symptoms; rather, it is intended to identify a heritable predisposition (i.e., latent variable) to diverse forms of disinhibitory psychopathology (e.g., Antisocial Personality Disorder, Conduct Disorder, Impulsivity, Low Constraint). Though there are many ways to measure psychopathy and externalizing traits, three common approaches use statistical and psychometric principles to parse these antisocial subtypes. First, diagnostic measures may be used to identify the unitary construct of psychopathy (Psychopathy Checklist-Revised) or diagnoses used as a proxy for latent trait externalizing (Antisocial Personality Disorder, Conduct Disorder). Second, different assessment measures of psychopathy (e.g., Psychopathy Checklist-Revised, Psychopathic Personality Inventory, Multidimensional Personality Questionnaire) and externalizing traits (Externalizing Spectrum Inventory; Multidimensional Personality Questionnaire) may be used to examine the impact of each subtype on the outcome of interest. Third, subscales of the Psychopathy Checklist-Revised, Factor1 (Interpersonal-Affective) and Factor2 (Impulsive-Antisocial) can be reliably used to identify individuals high on psychopathy or externalizing traits (see Methods). The studies included in the Introduction implement one, and in some cases multiple, of these strategies.
} 
individuals with psychopathy (Birbaumer et al., 2005; Patrick, Bradley, \& Lang, 1993), which investigators commonly attribute to a fundamental deficit in emotional reactivity. However, there is substantial evidence that these emotion deficits are moderated by experimental context. Newman and colleagues propose that this context specificity is associated with a core dysfunction in the adaptive deployment of selective attention that impedes information processing in individuals with psychopathy. More specifically, it is suggested that an early attention bottleneck filters and processes multi-dimensional information in serial, rather than simultaneously, thus hindering the processing of information that conflicts with goal-directed behavior (Baskin-Sommers, Curtin, \& Newman, 2011). For individuals with psychopathy, the bottleneck creates an advantage in many situations that require individuals to filter potential distracters (Hiatt, Schmitt, \& Newman, 2004; Mitchell, Richell, Leonard, \& Blair, 2006; Wolf et al., 2012; Zeier, Maxwell, \& Newman, 2009), but this advantage is counterbalanced by their reduced ability to attend to multiple ongoing streams of information (Baskin-Sommers, Curtin, \& Newman, 2013; Glass \& Newman, 2009; Newman \& Kosson, 1986). Consequently, this tradeoff results in a tendency to overlook important information unless it is directly related to their goal-directed focus of attention.

In support of this model, across diverse experimental paradigms, such as passive avoidance learning, instructed fear conditioning, moral decision-making, and picture-viewing, offenders with psychopathy display normal responses (e.g., behavioral inhibition, fearpotentiated startle, emotion-modulated startle, amygdala activation, and electrodermal activity) to affective information when it is part of their goal-directed task or embedded in a perceptually simple display (Baskin-Sommers et al., 2011, 2013; Dadds et al., 2006; Decety, Chen, Harenski, \& Kiehl, 2013; Meffert, Gazzola, den Boer, Bartels, \& Keysers, 2013; Newman, Curtin, Bertsch, \& Baskin-Sommers, 2010; Newman \& Kosson, 1986; Sadeh \& Verona, 2012). Yet their reactions to the same affective stimuli are deficient, relative to offenders without psychopathy, if their attention has been allocated to an alternative goal or complex aspect of the situation (see Newman \& Baskin-Sommers, 2011 for review). Combined, these studies show that affective and inhibitory deficits can appear and disappear in participants with psychopathy depending on whether or not affective or inhibitory information is congruent with their goal (Brazil et al., 2012; Glass \& Newman, 2009; Hiatt et al., 2004; Sadeh \& Verona, 2008, 2012). Functionally, this cognitive-affective deficit in attention to context, results in a myopic perspective on decision-making and goal-directed behavior, such that individuals with psychopathy are adept at using information that is directly relevant to their goal to effectively regulate behavior (e.g., modulate behavior and ignore emotions to con someone), but display impulsive behavior (e.g., quitting one's job in the absence of an alternative one) and egregious decision making (e.g., seeking publicity for a con while wanted by police) when information is beyond their immediate focus of attention.

By contrast, the behavior of individuals with externalizing traits reflects hyper-reactivity to emotional and other motivationally relevant cues, excessive reward seeking, intense hostility, and other strong urges that overwhelm inhibitory and cognitive controls. These individuals display exaggerated reactivity to affective stimuli (Baskin-Sommers, Wolf, 
Buckholtz, Warren, \& Newman, 2012; Frick \& Morris, 2004; Iacono, Malone, \& McGue, 2008; Mullin \& Hinshaw, 2007), have difficulty deploying executive functions (Endres, Rickert, Bogg, Lucas, \& Finn, 2011), and struggle to regulate their intense emotional reactions (Daughters, W, Kahler, Strong, \& Brown, 2005; Malterer, Glass, \& Newman, 2008). Increasing evidence suggests that though individuals with externalizing traits have a tendency to over-react to emotion information and display deficits in executive functions, these tendencies do not appear to impact behavior unless processing emotion and employing executive functions, such as cognitive control, is required (Baskin-Sommers et al., 2014; Sadeh et al., 2013). More specifically, it appears that individuals with externalizing traits are prone to over-allocate cognitive resources to potentially relevant stimuli in situations that foster an expectation that motivationally significant events will occur. And, moreover, that such over-allocation depletes resources available for processing subsequent stimuli and the implementation of capacity limited executive functions (e.g., inhibition, shifting, and control) that normally modulate ongoing behavior (Baskin-Sommers \& Newman, 2013).

This concomitant deficit in cognitive control and affective hyperreactivity is mirrored in the tendency for individuals with externalizing traits to demonstrate strong attentional orienting to salient reward cues (Avila \& Parcet, 2001), dysregulated responding in the presence of salient goal stimuli (Bachorowski \& Newman, 1990), an exaggerated deficiency in identifying secondary targets in the attentional blink task (Baskin-Sommers et al., 2012), failure to inhibit reward seeking responses, difficulty classifying rare or unexpected stimuli in the oddball task (Bernat, Nelson, Steele, Gehring, \& Patrick, 2011), and deficits in delay discounting during gambling tasks (Bobova, Finn, Rickert, \& Lucas, 2009). In each of these instances, individuals with externalizing traits react strongly to motivationally salient information (e.g., unexpected information, goal-relevant reward, punishment, threat), particularly when they are prepared to make a practiced, dominant response. Thus, individuals with externalizing traits may be disinhibited because of an inability to engage in cognitive control under affectively charged circumstances (Sadeh et al., 2013). This deficit in affective cognitive control leaves individuals with externalizing traits vulnerable to reactive behavior and a tendency to let dominant responses override cognitive control. According to Skeem and colleagues (2004) individuals with externalizing traits of this type may be described as "anxious, emotionally volatile, hostile, and impulsive, and they are heavy substance abusers" (p. 399). Accordingly, reactivity to affective information and deficiencies in executive function may enhance the ability of individuals with externalizing traits to engage in pleasure seeking (e.g., risky sexual behaviors, substance use) more wholeheartedly, display extraverted interpersonal tendencies (e.g., be outgoing or hotheaded), but also act in an impulsive manner, particularly when in an affectively charged situation (e.g., criminal activity, gambling, fight in reaction to a threat or insult).

Although psychopathy and externalizing traits both encompass antisocial behavior (e.g., aggression, substance abuse, impulsivity), there is increasing evidence that these antisocial subtypes are associated with distinct cognitive-affective deficits. More specifically, in the domains of emotion and executive functions, individuals with psychopathy versus externalizing traits often display remarkably different cognitive-affective functioning (see Baskin-Sommers \& Newman, 2013 for review). Whereas individuals with psychopathy are 
associated with an emotionally 'cold' style, individuals with externalizing traits are associated with an emotionally 'hot' style. For instance, using startle as an indirect measure of amygdala functioning, numerous studies find that individuals with psychopathy display reduced startle potentiation, but individuals with externalizing traits display excessive startle potentiation in affective picture-viewing and fear conditioning paradigms (Baskin-Sommers, Curtin \& Newman, 2012; Baskin-Sommers, Curtin, Larson, Stout, Kiehl \& Newman, 2012; Vaidyanathan et al., 2010). Similarly, individuals with psychopathy demonstrate superior distress tolerance, a measure of affective regulation, whereas individuals with externalizing traits demonstrate poor distress tolerance (Sargaent, Daughters, Curtin, Schuster \& Lejuez, 2011). With regard to executive functions, individuals with psychopathy do not display deficits in executive function tasks, and at times demonstrate superior executive functioning, whereas individuals with externalizing traits demonstrate poor cognitive control, conflict monitoring, and working memory (Blair, Newman, Mitchell, Richell, Leonard, Morton \& Blair, 2006; Dolan, Bechara \& Nathan, 2007; Endres, Rickert, Bogg, Lucas \& Finn, 2011; Morgan \& Lilienfeld, 2000). As a result of these differences in cognitive-affective functioning, progress in understanding and treating the serious behavior problems associated with psychopathy and externalizing traits depends upon disentangling and targeting these subtype-specific dysfunctions.

Building on the substantial scientific progress identifying and distinguishing the dysfunctional cognitive-affective processes associated with psychopathy and externalizing traits, the present study sought to develop novel cognitive remediation training that targets the processes associated with these two antisocial subtypes. That is, with appropriate training, individuals with psychopathy who are normally oblivious to important affective, inhibitory, and punishment cues that contraindicate ongoing goal-directed behavior may learn to attend to context, notice important interpersonal and situational cues and changes in their environment. Conversely, individuals with externalizing traits may learn to engage affective cognitive control by acting rather than over-reacting to affective information such as insults and other motivationally salient information like monetary gains, and, thus, avoid depletion of their executive function capabilities.

\section{Methods}

Participants

One hundred and forty-one participants were screened for eligibility. A prescreen of institutional files and assessment materials were used to exclude individuals who performed below the fourth-grade level on a standardized measure of reading or math achievement, who scored below 70 on a brief measure of IQ (Zachary, 1986), or who had diagnoses of schizophrenia, bipolar disorder, or psychosis, not otherwise specified. The intelligence cutoff and exclusion of major psychopathology were used primarily to reduce the contribution of these extraneous influences on the assessment of performance. Additionally, all participants were between the ages of 18 and 45 because antisocial behavior has been found to change with advancing age (Hare et al., 1990; Steffensmeier, Allan, Harer \& Streifel, 1989). After the initial assessment, 124 eligible male offenders with psychopathy or externalizing traits were randomly assigned to one of two trainings (see Online Supplement 
Fig. 1 for CONSORT diagram). An additional 21 participants were excluded due to institutional transfer or aberrant performance data (i.e., regression outliers based on studentized residuals Bonferroni-corrected p-values <.05). The final sample consisted of 103 inmates. All participants provided written informed consent according to the procedures set forth by the University of Wisconsin-Madison Human Subjects Insitutional Review Board. Participants were also informed that their decision to take part in the project or to refuse would have no influence on their status within the correctional system.

All participants were assessed using file information and a semi-structured interview that lasted approximately 60 minutes and provided sufficient information to diagnose psychopathy and externalizing traits using the Psychopathy Checklist-Revised (PCL-R) (Hare, 2003). The PCL-R is considered the gold-standard measure of antisocial subtypes/ psychopathy, particularly with incarcerated samples. This measure contains 20 items that are rated 0,1 , or 2 according to the degree to which a characteristic is present: significantly (2), moderately (1), or not at all (0) and identifies individuals displaying a combination of disinhibited traits (i.e., impulsivity, irresponsibility), a chronic antisocial lifestyle, and a variety of interpersonal and affective symptoms (i.e., callousness, glibness, superficial charm, shallow emotions).

Factor analytic studies of the PCL-R have revealed a variety of factor solutions, but many studies of psychopathic behavior employ the two-factor model owing to the relatively reliable and distinct correlates associated with these two factors (Harpur, Hakstien \& Hare, 1989; Hare, 2003). Whereas PCL-R Factor2 taps the impulsive and chronic antisocial tendencies that are common to most antisocial subtypes, the Factor1 items tap the interpersonal (charm, grandiosity, and deceitfulness/conning) and affective (lack of remorse, empathy, and emotional depth) features that distinguish psychopathy from other antisocial subtypes. Of particular relevance to this study, a wealth of evidence serves to link Factor2 with externalizing traits and diagnoses. For instance, individuals with high PCL-R Factor2 scores display similar symptoms and external correlates as individuals with Antisocial Personality Disorder, high levels of externalizing traits, and low constraint (Patrick, 1993; Patrick, 2007; Patrick et al., 2008; Vaidyanathan et al., 2010). Direct investigations of the association between PCL-R Factor2 symptoms and the externalizing dimension demonstrate a high degree of correspondence, especially when controlling for PCL-R Factor1 scores (e.g., Patrick, Hicks, Krueger \& Lang, 2005). In addition, there is substantial evidence that the cognitive-affective correlates associated with the unique variance of PCL-R Factor2 resemble those associated with externalizing traits and diagnoses and, moreover, differ predictably from those associated with PCL-R Factor 1 and PCL-R Total scores (e.g., Baskin-Sommers, Zeier \& Newman, 2009; Patrick, 2007).

Based on this evidence, we grouped participants using the PCL-R factor scores to distinguish psychopathic and externalizing subtypes (see also Patrick, 1993). More specifically, we identified participants who scored a twelve or above on impulsive-antisocial (Factor2) items and then divided them into two subgroups using the sample median for interpersonal-affective (Factor1) items. Given that psychopathy is classically defined as the combination of interpersonal-affective (i.e., Factor1) and impulsive-antisocial (i.e., Factor2) traits, participants who scored a twelve or above on Factor2 and above the median on 
Factor 1 items were assigned to the psychopathy group. Participants who scored a twelve or above on Factor2, but below the median on Factor1 items were assigned to the externalizing group.

\section{Procedure}

Briefly, prior to testing or training, all participants completed an assessment session. Initially participant eligibility was established using a life-history interview, the Psychopathy Checklist-Revised (Hare, 2003), and institutional files to establish their appropriate subtypebased group. Using this information, all eligible participants were randomly assigned to one of two cognitive remediation trainings, with the constraint that participants were equally likely to be drawn from the psychopathy and externalizing groups. Moreover, within antisocial subtype, participants assigned to the two trainings were matched with regard to age, IQ, and ethnicity (see Supplemental Fig. 1). Matching for the cognitive remediation training groups was conducted using a nearest neighbor method.

At least one week after the assessment session, all inmates, regardless of training assignment, completed a battery of behavioral (e.g., accuracy and reaction time) and psychophysiological (e.g., fear-potentiated startle and event-related potential) assessments, over two sessions that evaluated the cognitive-affective deficits typically associated with these antisocial subtypes. This battery included five tasks that have been previously used to tap and distinguish the cognitive-affective processes associated with psychopathy and externalizing traits (see Online Supplement). Three of the tasks, instructed fear conditioning, modified stroop, and lexical decision, primarily evaluate the attention to context deficit present in individuals with psychopathy. Consistent with their limited processing of context, previous research indicates that individuals with psychopathy: (1) display deficient fear potentiated startle, particularly when attention is engaged prior to presentation of the threatrelevant information (Baskin-Sommers et al., 2011), (2) display less interference to distracting contextual information on modified stroop tasks (e.g., Hiatt et al., 2004), and (3) display less responsiveness to the task-irrelevant, emotion connotations of the word, and thus less emotion facilitation on lexical decision tasks (Lorenz \& Newman, 2002a, 2002b; Williamson, Harpur \& Hare, 1991). Conversely, externlizing is unrelated to these three performance dysfunctions (Baskin-Sommers, Curtin, Larson, Stout, Kiehl \& Newman, 2012; Hiatt et al., 2004; Lorenz \& Newman, 2002b). Two of the tasks, $n$-back and Paced Auditory Serial Addition Task-Computerized, are purported to measure principally the affective cognitive control deficits present in individuals with externalizing traits. Related to their difficulty balancing the demands on affective processing and executive functioning, previous research indicates that individuals with externalizing traits: (1) perform poorly during the $n$-back on trials that place demands on cognitive control and provide incentives for performance (Baskin-Sommers et al., 2014) and (2) display poor distress tolerance on the Paced Auditory Serial Addition Task (Lejuez, Kahler, \& Brown, 2003; Sargaent et al., 2011). By contrast, there is no evidence that individuals with psychopathy show similar deficits on these tasks (e.g., Sargaent et al., 2011). Therefore, each of these pre-post tasks was selected to capture the unique cognitive-affective deficits associated with each of the antisocial subtypes. 
One week following the pre-training assessment sessions, inmates began a once a week, hour per session, computerized training program for six consecutive weeks. This study employed a fully crossed $2 \times 2$ design, where half of the inmates received cognitive remediation training ( 3 computerized tasks per training) that matched their specific cognitive-affective deficit and half received a treatment that did not match their deficit (but matched the deficit of the other subtype of antisocial offender). As such, this design included two active treatments and two antisocial subtypes with distinct treatment needs to evaluate the specificity of change. Addressing the psychopathic-specific deficit, attention to context (ATC) training focused on learning to attend to and integrate contextual cues present in the environment. Three tasks, reversal learning (Budhani, Richell, \& Blair, 2006), divided visual field (Kosson, 1998; Llanes \& Kosson, 2006), and affective gaze (Baskin-Sommers \& Newman, 2014), require ATC functioning and provide individuals with practice noticing changes in contextual information, such as rule changes and using emotion information to modulate behavior. By contrast, affective cognitive control (ACC) training was designed to address the externalizing-specific deficit and provide individuals with practice inhibiting behavior, particularly under motivational or affective contexts. Three tasks, breath holding (Sütterlin et al., 2013), incentivized Go-Stop (Albrecht, Banaschewski, Brandeis, Heinrich, \& Rothenberger, 2005; Avila \& Parcet, 2001; Schuckit et al., 2012), and Simon (Lu \& Proctor, 1995; Simon \& Rudell, 1967), tap ACC functioning and place demands on the basic employment of cognitive control, such as task switching, as well as on the concurrent engagement of cognitive control and affective processing (e.g., performing a task in the face of distress [uncomfortable feelings] or motivating cues [monetary incentives]). Rather than training performance on a particular task as done in previous cognitive remediation research, in both training programs, we attempted to develop a more broad-spectrum and generalizable skill by using three different, albeit conceptually related, tasks to address each cognitive-affective deficit.

One week after the end of the six-week training period, inmates completed a post-training assessment battery, over two sessions, that was identical to the one that was administered pre-training. The pre/post tasks and the training tasks were conceptually related, but constituted distinct assessments of the cognitive-affective deficits operating in these antisocial subtypes (Fig. 1). Use of these pre-post assessments provided a means to assess whether that the effects of training transfers (i.e., generalizes) to untrained tasks, and moreover, that training can impact a network of related cognitive abilities. Overall, by using multiple measures of each construct and explicitly evaluating their effects within the $2 \times 2$ design, we are able to rigorously define the key cognitive-affective processes, control for non-specific treatment effects, and specify mechanism-specific change.

\section{Data Analysis}

Analysis of training and pre-post measures was performed separately and occurred in multiple stages. First, for both training and pre-post tasks, representative measures (see Online Supplement) from each task were extracted. Second, change scores were calculated. For each training task, a change score over the 6 sessions of training was calculated using within-subject regression, such that each of the training data points was regressed on session number and higher beta values represent greater change over training. Within the pre-post 
tasks, change scores were calculated by subtracting scores at pre from scores at post, for each pre-post measure, such that higher numbers always represent improvement in performance. Third, separate repeated measures analysis of variance (ANOVA) models were run for the ATC training ( 3 tasks $\times 2$ antisocial subtypes), ACC training ( 3 tasks $\times 2$ antisocial subtypes), and pre-post tasks (5 tasks $\times 2$ antisocial subtypes $\times 2$ training types). For the two training programs and pre-post assessments, preliminary ANOVAs revealed no interactions of task with group or training type, but a significant between-group main effect $(p<.05)$. Therefore, for each training program and the five task pre-post assessment, we collapsed across task and calculated a standardized (z-score) average change score. These standardized averages of change are the measures presented in the primary results reported below. Finally, despite the statistical justification for combining the pre-post measures, these tasks were selected a priori as indicators of psychopathy ( 3 tasks) and externalizing ( 2 tasks) deficits, respectively. Therefore, following the aggregate pre-post analyses, we present the more specific analyses that focus separately on the psychopathy-related and externalizingrelated pre-post tasks. Partial eta square $\left(\eta_{\mathrm{p}}{ }^{2}\right)$ values are reported as an index of effect size.

\section{Results}

The results demonstrated the potential of using cognitive remediation training to bring about subtype-specific improvement in dysfunctional cognitive-affective mechanisms. First, using linear regression, we examined the relationship between antisocial subtype and change in cognitive performance over the six-week training period. A significant two-way interaction between antisocial subtype (Psychopathy, Externalizing) and training type (ATC, ACC) $\left(F(1,99)=13.16, p<.001, \eta_{\mathrm{p}}{ }^{2}=.12\right)$ indicated that only the deficit-matched antisocial subtype displayed significant improvement in training task performance over time (Fig. 2). That is, individuals with psychopathy improved significantly $(\mathrm{p}<.01)$ during ATC training $(\mathrm{M}=.45$, $\mathrm{SE}=.16, \eta_{\mathrm{p}}{ }^{2}=.26$ ), which was developed to target their cognitive-affective deficit, whereas individuals with psychopathy who received the non-deficit matched ACC training did not improve with training $\left(\mathrm{M}=-.33, \mathrm{SE}=.16, \eta_{\mathrm{p}}{ }^{2}=.16\right)$. Conversely, individuals with externalizing traits demonstrated significant $(\mathrm{p}=.05)$ improvement during ACC training $\left(\mathrm{M}=.20, \mathrm{SE}=.17, \eta_{\mathrm{p}}{ }^{2}=.02\right)$, which was designed to address their cognitive-affective deficit, but failed to improve during ATC training $\left(\mathrm{M}=-.28, \mathrm{SE}=.18, \eta_{\mathrm{p}}{ }^{2}=.11\right)$.

Next we examined the generalizability of this training to a battery of pre-post tasks selected to assess the cognitive-affective deficits associated with these antisocial subtypes. Examination of the pre-post tasks revealed a significant two-way interaction (Training type $\mathrm{x}$ Antisocial Subtype), demonstrating that individuals who received training that matched their cognitive-affective deficit not only improved on trainings but also demonstrated significant improvement across all five of the pre-post measures $(F(1,99)=12.87, p<.001$, $\eta_{\mathrm{p}}{ }^{2}=.12$ ) (Fig. 3). Examination of the within-antisocial subtype simple effects revealed that individuals with psychopathy who received ATC training displayed significantly $(\mathrm{p}<.01)$ greater improvement on the pre-post battery $\left(\mathrm{M}=.31, \mathrm{SE}=.14, \eta_{\mathrm{p}}{ }^{2}=.21\right)$ than individuals with psychopathy who received ACC training ( $\left.\mathrm{M}=.-15, \mathrm{SE}=.14, \eta_{\mathrm{p}}^{2}=.04\right)$. Conversely, individuals with externalizing traits who received ACC training displayed significantly ( $\mathrm{p}=$. 02) greater improvement on the pre-post battery $\left(\mathrm{M}=.19, \mathrm{SE}=.15, \eta_{\mathrm{p}}{ }^{2}=.05\right)$ than individuals with externalizing traits who received training in $\operatorname{ATC}\left(\mathrm{M}=-.43, \mathrm{SE}=.16, \eta_{\mathrm{p}}{ }^{2}=.23\right)$. Taken 
together, these results indicate that the individuals with psychopathy and externalizing traits are capable of overcoming their subtype-specific deficits with practice and that receiving deficit-matched training results in generalizable change in these subtype-specific deficits.

Although preliminary analyses indicated that there was no interaction among the five prepost tasks, three of the pre-post measures were selected, a priori, to assess the psychopathyrelated dysfunction in ATC whereas two of them primarily assessed the externalizing-related dysfunction in ACC. Therefore, secondary analyses were conducted to examine the generalizability of training to the group of tasks related to the psychopathy dysfunction and the group of tasks related to the externalizing dysfunction in two separate ANOVAs.

Consistent with the primary analysis, within the psychopathy-related pre-post measures, a significant two-way interaction between antisocial subtype (Psychopathy, Externalizing) and training type (ATC, ACC) $\left(F(1,99)=11.20, p<.001, \eta_{\mathrm{p}}{ }^{2}=.10\right)$ indicated that only the deficitmatched antisocial subtype displayed significant improvement in pre-post performance over time. Examination of the simple effects revealed that individuals with psychopathy who received the ATC training $\left(\mathrm{M}=.42, \mathrm{SE}=.18, \eta_{\mathrm{p}}{ }^{2}=.14\right)$ displayed significantly $(\mathrm{p}=.01)$ more change on the psychopathy-related pre-post measure than those who received the ACC training $\left(\mathrm{M}=-.12, \mathrm{SE}=.14, \eta_{\mathrm{p}}{ }^{2}=.03\right)$. With regard to the externalizing-related pre-post measures, a significant two-way interaction between antisocial subtype (Psychopathy, Externalizing) and training type (ATC, ACC) $\left(F(1,99)=4.97, p=.03, \eta_{\mathrm{p}}{ }^{2}=.05\right)$ indicated that only the deficit-matched antisocial subtype (i.e., individuals with externalizing traits versus psychopathy) displayed improvement in pre-post performance over time. However, comparison of individuals within antisocial subtype, indicated that individuals with externalizing traits who received a deficit-matched ACC training $\left(\mathrm{M}=.22, \mathrm{SE}=.23, \eta_{\mathrm{p}}{ }^{2}=.04\right)$ did not display significantly $(\mathrm{p}=.15)$ more change from pre to post than individuals with externalizing traits who received a non-deficit-matched ATC training $(\mathrm{M}=-.27, \mathrm{SE}=.15$, $\left.\eta_{\mathrm{p}}^{2}=.13\right)$.

\section{Discussion}

This study compared novel cognitive remediation training programs for individuals with psychopathy and externalizing traits. Results demonstrate that training designed to remedy the distinct deficits of these two antisocial subtypes resulted in differential improvement on both trained and non-trained (pre-post) tasks. Moreover, these effects were specific to the group who received deficit-matched training. Research in psychopathology proceeds on the assumption that identification of core cognitive-affective mechanisms that predispose and/or maintain psychopathology will ultimately result in significantly improved treatment and prevention of problematic behavior. Yet, to date, evidence for the successful translation of such progress into specific and meaningful interventions is surprisingly limited (Wampold, 2007). This paradox is particularly evident in the treatment of antisocial psychopathology. Although antisocial individuals are relatively resistant to traditional therapies, advances in knowledge concerning their cognitive-affective deficits, and recent progress in cognitive remediation, highlight new treatment options for addressing their costly criminal behavior and chronic substance abuse. The present study represents a major step in identifying, developing, and evaluating mechanism-based interventions for these individuals.

Clin Psychol Sci. Author manuscript; available in PMC 2016 January 01. 
Consistent with hypotheses, individuals with psychopathy who received the deficit-matched training improved on the ATC-specific training tasks. Additionally, their significant improvement on a separate group of laboratory measures indicated that they mastered a generalizable cognitive skill. Given explicit practice and skill building in balancing attention between primary and peripheral information, individuals with psychopathy became more responsive to an array of affective and non-affective information on an aggregate measure of cognitive-affective functioning and on specific measures that more directly tapped the psychopathy-related dysfunction. Individuals with psychopathy have long been considered among the most dangerous and difficult to treat individuals, however, the present results suggest that their cognitive-affective dysfunctions may be trained and rehabilitated.

Individuals with externalizing traits who received appropriate training also improved, demonstrating an enhanced ability to act rather than over-react to affective and motivationally salient information and, thus, avoid depletion of their executive function capabilities. More specifically, individuals with externalizing traits displayed significant improvement on the training tasks. However, evidence for the relative superiority of ACC over ATC training in yielding generalizable change (i.e., pre-post), particularly on tasks purported to tap primarily ACC functioning, was limited. Even though individuals with externalizing traits who received ACC training descriptively showed improvement on the pre-post measures, the significance of the interaction was largely attributable to the degraded post-task performance of individuals with externalizing traits who completed the ATC training. That is, individuals with externalizing traits who received ATC training appeared to exhibit iatrogenic effects of treatment, suggesting that receiving a treatment unrelated to their deficit may actually exacerbate their hyperreactivity to negative events or feedback (see also, Pardini, Lochman, \& Powell, 2007; Pasalich, Dadds, Hawes, \& Brennan, 2011).

Given the intractable nature of their deficits and long-standing pessimism about treating antisocial subtypes, these results, particularly those for individuals with psychopathy, highlight the substantial potential for addressing the disinhibited and costly behavior of antisocial individuals by identifying and targeting their specific cognitive-affective deficits. The promise of basic research in experimental psychopathology is to identify specific dysfunctional processes that may be used to treat and prevent costly clinical syndromes. Armed with such information, it is possible to employ powerful research designs to advance the technology of clinical interventions. Specifically, once identified, investigators can measure the dysfunctional process, manipulate it through intervention, measure the predicted change, and examine the extent to which change in the putative deficits yields desired changes in psychological and brain functioning and, more importantly, in clinical outcomes. In contrast to generic and static interventions, this scientific approach promotes the initiation, personalization, and maintenance of behavior change by integrating work across theoretical and methodological domains. Of course, though, more work is needed to increase the focus and depth at each level of treatment development, from the conceptualization of the phenotype to the prediction of clinical outcomes.

A significant challenge to developing deficit-specific interventions relates to the specification of the phenotype and selection of representative measures. For decades, research on psychopathy has benefitted from the existence of a well-validated and widely 
used assessment of the syndrome (viz. the PCL-R). In turn, the associations between the PCL-R, particularly high scores of both Factor1 and Factor2, and specific process-relevant laboratory measures are well established. By contrast, the study of externalizing is less developed, in that there is no widely agreed upon measure of trait externalizing and, thus, the evidence linking any specific measure of externalizing traits with particular measures of cognitive-affective dysfunction is less well established ${ }^{2}$. It is possible that, relative to psychopathy, the externalizing effects in the present study demonstrate less specificity because these traits were measured using a scale that overlaps with psychopathy (i.e., PCL$\mathrm{R}$ Factor2 versus a more distinctive index of externalizing such as low constraint) or that the assessment of the mechanism requires further refinement. Given the heterogeneity of antisocial subtypes, more work is needed to identify more powerful methods for characterizing individuals with relatively distinct and homogeneous cognitive-affective deficits. In this way, there is the potential to develop a richer and incremental science of behavior change that links increasingly specific cognitive-affective problems to increasingly specific interventions in service of developing increasingly efficient and efficacious interventions.

In addition to refining the conceptualization of particular antisocial traits and methods for assessing those traits and trait-based deficits, it is essential for this type of translational research to extend into the crucial domain of real-world behaviors. In the present study, change from pre to post-training is the only measure of generalizability beyond the training tasks. Therefore, it is unclear whether training will reduce real-world behaviors, such as criminal activity, substance abuse, and risky sexual activity. Though the present study is unable to measure the link between laboratory performance and real-world behavior change, there is reason to believe that addressing the functional components of a deficit in ATC or ACC has the potential to bring about clinically meaningful behavior change. For example, training children with callous-unemotional traits (psychopathy) on the perception and interpretation of human emotions (i.e., noticing contextual cues) improves empathic functioning (Dadds, Cauchi Wimalaweera, Hawes \& Brennan, 2012). Similarly, practicing distress tolerance improves treatment outcome and the quality of distress tolerance predicts the ability to maintain abstinence in individuals with substance use disorders (Bornovolova, Gratz, Daughters, Hunt \& Lejuez, 2012; Daughters, Lejuez, Kahler, Strong \& Brown, 2005). Further, the concept of neuro-prediction suggests that identification of specific neural (dys)functions may help quantify an individual's potential responsiveness to treatment (see McGrath et al., 2013 for an example in depression) and the likelihood of engaging in important clinically relevant behaviors (see Aharoni et al., 2013 for an example in prisoners). Thus, the combination of identifying the functional and neuroanatomical components of deficits in ATC and ACC and using that information to not only target, but also predict, behavior change as a result of treatment is an extraordinarily exciting possibility. Using increasingly specific indicators of dysfunctional processes to predict the differential efficacy of particular training strategies represents a powerful methodology for

\footnotetext{
${ }^{2}$ A notable exception to this statement is the well-developed association between the latent trait externalizing and smaller P3 responses in the oddball task. However, because there is less evidence that the oddball differentiates psychopathy and externalizing (Kiehl et al., 1999), it was not well suited as a specific training/pre-post assessment of affective cognitive control.
} 
continually testing and revising assumptions about the core deficit and refining interventions to optimize desired changes in behavior.

In sum, the current results presage a new era of developing specific remediation training regimes to target the cognitive-affective dysfunctions that subvert behavioral control and result in major psychopathology. For decades, mental health professionals have decried the patient "uniformity myth" (Kiesler, 1966) and advocated for an individualized approach to clinical interventions (e.g., Project MATCH)(Mattson \& Allen, 1991). Unfortunately, investigators have had surprisingly little success in accommodating person-specific dysfunction in treatment research and clinical practice. Perhaps one of the central limitations of previous efforts relates to the failure to integrate definitive research on basic mechanisms with broader treatment development. Ultimately, the success of individualized medicine requires a higher-level integration of these disciplines. The current findings highlight the potential for utilizing a conceptual and multilevel methodological framework to connect particular cognitive-affective mechanisms to the hypothesized action of effective treatments. The present results offer promise for changing neural and behavioral patterns, even for what many consider to be the most recalcitrant treatment population.

\section{Supplementary Material}

Refer to Web version on PubMed Central for supplementary material.

\section{Acknowledgments}

This work was supported by grant R21DA030876 from NIDA. We wish to thank the Wisconsin Department of Corrections, and especially Dr. Kevin Kallas and Warden Tom Nickel, for their continued support of this research program.

\section{References}

Aharoni E, Vincent GM, Harenski C, Calhoun VD, Sinnott-Armstrong W, et al. Neuroprediction of future rearrest. PNAS. 2013; 110:6223-6228. [PubMed: 23536303]

Albrecht B, Banaschewski T, Brandeis D, Heinrich H, Rothenberger A. Response inhibition deficits in externalizing child psychiatric disorders: An ERP-study with the Stop-task. Behavioral and Brain Functions. 2005; 1(1):22. [PubMed: 16336676]

Avila C, Parcet MA. Personality and inhibitory deficits in the stop-signal task: the mediating role of Gray's anxiety and impulsivity. Personality and Individual Differences. 2001; 31(6):975-986.

Bachorowski J, Newman J. Impulsive motor behavior: Effects of personality and goal salience. Journal of Personality and Social Psychology. 1990; 58(3):512-518. [PubMed: 2324940]

Barbaree HE. Psychopathy, treatment behavior, and recidivism: an extended follow-up of Seto and Barbaree. Journal of Interpersonal Violence. 2005; 20(9):1115-1131. [PubMed: 16051730]

Baskin-Sommers AR, Zeier JD, Newman JP. Self-Reported Attentional Control Differentiates the Major Factors of Psychopathy. Personality and Individual Differences. 2009; 47:626-630. PMC2789461. [PubMed: 19997525]

Baskin-Sommers A, Curtin JJ, Newman J. Specifying the Attentional Selection That Moderates the Fearlessness of Psychopathic Offenders. Psychological Science. 2011; 22(2):226-234. [PubMed: 21245494]

Baskin-Sommers AR, Curtin JJ, Newman JP. Specifying the attentional selection that moderates the fearlessness of psychopathic offenders. Psychological Science. 2011; 22:226-234. PMC3358698. [PubMed: 21245494] 
Baskin-Sommers A, Curtin JJ, Newman J. Emotion-modulated startle in psychopathy: Clarifying familiar effects. Journal of Abnormal Psychology. 2013; 122(2):458-468. [PubMed: 23356218]

Baskin-Sommers A, Krusemark EA, Curtin JJ, Lee C, Vujnovich A, Newman J. The impact of cognitive control, incentives, and working memory load on the $\mathrm{P} 3$ responses of externalizing prisoners. Biological Psychology. 2014; 96:86-93. [PubMed: 24355244]

Baskin-Sommers, A.; Newman, J. Differentiating the Cognition-Emotion Interactions that Characterize Psychopathy versus Externalizing Disorders. In: Robinson, MD.; Watkins, ER.; Harmon-Jones, E., editors. Handbook of Cognition and Emotion. New York, NY, US: Guilford Press; 2013. p. 501-520.

Baskin-Sommers AR, Curtin JJ, Larson CL, Stout D, Kiehl KA, Newman JP. Characterizing the Anomalous Cognition-Emotion Interactions in Externalizing. Biological Psychology. 2012; 91:48-58. PMC3407296. [PubMed: 22579718]

Baskin-Sommers A, Wolf R, Buckholtz J, Warren C, Newman J. Exaggerated Attention Blink Response in Prisoners with Externalizing. Journal of Research in Personality. 2012; 46(6):688693. [PubMed: 23180900]

Bernat EM, Nelson LD, Steele VR, Gehring WJ, Patrick CJ. Externalizing psychopathology and gainloss feedback in a simulated gambling task: dissociable components of brain response revealed by time-frequency analysis. Journal of Abnormal Psychology. 2011; 120(2):352-364. [PubMed: 21319875]

Birbaumer N, Veit R, Lotze M, Erb M, Hermann C, Grodd W, Flor H. Deficient fear conditioning in psychopathy: a functional magnetic resonance imaging study. Archives of General Psychiatry. 2005; 62(7):799-805. [PubMed: 15997022]

Blair KS, Newman C, Mitchell DGV, Richell RA, Leonard A, Morton J, Blair RJR. Differentiating among prefrontal substrates in psychopathy: Neuropsychological test findings. Neuropsychology. 2006; 20:153-165. [PubMed: 16594776]

Bobova L, Finn PR, Rickert ME, Lucas J. Disinhibitory Psychopathology and Delay Discounting in Alcohol Dependence: Personality and Cognitive Correlates. Experimental and Clinical Psychopharmacology. 2009; 17(1):51-61. [PubMed: 19186934]

Bornovalova MA, Gratz KL, Daughters SB, Hunt ED, Lejuez CW. Initial RCT of a distress tolerance treatment for individuals with substance use disorders. Drug and Alcohol Dependence. 2012; 122(1-2):70-76. [PubMed: 21983476]

Brazil IA, Verkes RJ, Brouns BHJ, Buitelaar JK, Bulten BH, de Bruijn ERA. Differentiating Psychopathy from General Antisociality Using the P3 as a Psychophysiological Correlate of Attentional Allocation. PLoS ONE. 2012; 7(11):e50339. [PubMed: 23166843]

Budhani S, Richell RA, Blair RJR. Impaired reversal but intact acquisition: Probabilistic response reversal deficits in adult individuals with psychopathy. Journal of Abnormal Psychology. 2006; 115(3):552-558. [PubMed: 16866595]

Compton WM 3rd, Cottler LB, Jacobs JL, Ben-Abdallah A, Spitznagel EL. The role of psychiatric disorders in predicting drug dependence treatment outcomes. The American Journal of Psychiatry. 2003; 160(5):890-895. [PubMed: 12727692]

Dadds MR, Cauchi AJ, Wimalaweera S, Hawes DJ, Brennan J. Outcomes, moderators, and mediators of empathic-emotion recognition training for complex conduct problems in childhood. Psychiatry Research. 2012; 199(3):201-207. [PubMed: 22703720]

Dadds MR, Perry Y, Hawes DJ, Merz S, Riddell AC, Haines DJ, ... Abeygunawardane AI. Attention to the eyes and fear-recognition deficits in child psychopathy. The British Journal of Psychiatry. 2006; 189(3):280-281. [PubMed: 16946366]

Daughters SB, W C, Kahler CW, Strong DR, Brown RA. Psychological Distress Tolerance and Duration of Most Recent Abstinence Attempt Among Residential Treatment-Seeking Substance Abusers. Psychology of Addictive Behaviors. 2005; 19(2):208-211. [PubMed: 16011392]

Daughters SB, Lejuez CW, Bornovalova MA, Kahler CW, Strong DR, Brown RA. Distress tolerance as a predictor of early treatment dropout in a residential substance abuse treatment facility. Journal of Abnormal Psychology. 2005; 114(4):729of. [PubMed: 16351393] 
Decety J, Chen C, Harenski C, Kiehl KA. An fMRI study of affective perspective taking in individuals with psychopathy: imagining another in pain does not evoke empathy. Frontiers in Human Neuroscience. 2013; 7

Dolan SL, Bechara A, Nathan PE. Executive dysfunction as a risk marker for substance abuse: the role of impulsive personality traits. Behavioral Sciences and the Law. 2008; 26:799-822. [PubMed: 19039793]

Endres MJ, Rickert ME, Bogg T, Lucas J, Finn PR. Externalizing psychopathology and behavioral disinhibition: Working memory mediates signal discriminability and reinforcement moderates response bias in approach-avoidance learning. Journal of Abnormal Psychology. 2011; 120(2): 336-351. [PubMed: 21381806]

Frick PJ, Morris AS. Temperament and developmental pathways to conduct problems. Journal of Clinical Child and Adolescent Psychology: The Official Journal for the Society of Clinical Child and Adolescent Psychology, American Psychological Association, Division 53. 2004; 33(1):5468.

Glass SJ, Newman J. Emotion processing in the criminal psychopath: the role of attention in emotionfacilitated memory. Journal of Abnormal Psychology. 2009; 118(1):229-234. [PubMed: 19222329]

Hare, RD. Manual for the Revised Psychopathy Checklist. 2nd ed.. Toronto, Ontario, Canada: MultiHealth Systems; 2003.

Hare RD. Psychopathy: a clinical and forensic overview. The Psychiatric Clinics of North America. 2006; 29(3):709-724. [PubMed: 16904507]

Hare RD, Harpur TJ, R A, Forth AE, Hart SD, Newman J. The revised Psychopathy Checklist: Reliability and factor structure. Psychological Assessment: A Journal of Consulting and Clinical Psychology. 1990; 2(3):338-341.

Harpur TJ, Hare RD, Hakstian AR. Two-factor conceptualization of psychopathy: Construct validity and assessment implications. Psychological Assessment. 1989; 1(1):6-17.

Hiatt KD, Schmitt WA, Newman J. Stroop Tasks Reveal Abnormal Selective Attention Among Psychopathic Offenders. Neuropsychology. 2004; 18(1):50-59. [PubMed: 14744187]

Hicks BM, Markon KE, Patrick CJ, Krueger RF, Newman J. Identifying Psychopathy Subtypes on the Basis of Personality Structure. Psychological Assessment. 2004; 16(3):276-288. [PubMed: 15456383]

Iacono WG, Malone SM, McGue M. Behavioral Disinhibition and the Development of Early-Onset Addiction: Common and Specific Influences. Annual Review of Clinical Psychology. 2008; 4(1): 325-348.

Kiehl KA, Hare RD, Liddle PF, McDonald JJ. Reduced P300 responses in criminal psychopaths during a visual oddball task. Biological Psychiatry. 1999; 11:1498-1507. [PubMed: 10356633]

Kiesler DJ. Some myths of psychotherapy research and the search for a paradigm. Psychological Bulletin. 1966; 65:110-136.

Klingberg T. Training and plasticity of working memory. Trends in Cognitive Sciences. 2010; 14(7): 317-324. [PubMed: 20630350]

Kosson DS. Divided visual attention in psychopathic and nonpsychopathic offenders. Personality and Individual Differences. 1998; 24(3):373-391.

Lejuez CW, Kahler CW, Brown RA. A modified computer version of the Paced Auditory Serial Addition Task (PASAT) as a laboratory-based stressor. The Behavior Therapist. 2003; 26(4):290 293.

Llanes SJ, Kosson DS. Divided Visual Attention and Left Hemisphere Activation Among Psychopathic and Nonpsychopathic Offenders. Journal of Psychopathology and Behavioral Assessment. 2006; 28(1):9-18.

Lorenz AR, Newman J. Deficient response modulation and emotion processing in low-anxious Caucasian psychopathic offenders: results from a lexical decision task. Emotion (Washington, D.C.). 2002a; 2(2):91-104.

Lorenz AR, Newman J. Utilization of emotion cues in male and female offenders with antisocial personality disorder: results from a lexical decision task. Journal of Abnormal Psychology. 2002b; 111(3):513-516. [PubMed: 12150427] 
Lu C, Proctor RW. The influence of irrelevant location information on performance: A review of the Simon and spatial Stroop effects. Psychonomic Bulletin \& Review. 1995; 2(2):174-207. [PubMed: 24203654]

Malterer MB, Glass SJ, Newman J. Psychopathy and Trait Emotional Intelligence. Personality and Individual Differences. 2008; 44(3):735-745. [PubMed: 18438451]

Mattson ME, Allen JP. Research on matching alcoholic patients to treatments: findings, issues, and implications. Journal of Addictive Diseases. 1991; 11(2):33-49. [PubMed: 1811759]

McGrath CL, Kelley MD, Holzheimer III PE, Dunlop BW, Craighead WE, et al. Toward a neuroimaging treatment selection biomarker for major depressive disorder. JAMA Psychiatry. 2013; 70:821-829. [PubMed: 23760393]

Meffert H, Gazzola V, den Boer JA, Bartels AAJ, Keysers C. Reduced pontaneous but relatively normal deliberate vicarious representations in psychopathy. Brain. 2013; 136(8):2550-2562. [PubMed: 23884812]

Mitchell DGV, Richell RA, Leonard A, Blair RJR. Emotion at the expense of cognition: psychopathic individuals outperform controls on an operant response task. Journal of Abnormal Psychology. 2006; 115(3):559-566. [PubMed: 16866596]

Mullin, BC.; Hinshaw, SP. Handbook of emotion regulation. New York, NY, US: Guilford Press; 2007. Emotion Regulation and Externalizing Disorders in Children and Adolescents; p. 523-541.

Newman, J.; Baskin-Sommers, A. Early Selective Attention Abnormalities in Psychopathy: Implications for Self-Regulation. In: Posner, M., editor. Cognitive Neuroscience of Attention. Second. New York, NY, US: Guilford Press; 2011. p. 421-440.

Newman J, Curtin JJ, Bertsch JD, Baskin-Sommers AR. Attention moderates the fearlessness of psychopathic offenders. Biological Psychiatry. 2010; 67(1):66-70. [PubMed: 19793581]

Newman J, Kosson DS. Passive avoidance learning in psychopathic and nonpsychopathic offenders. Journal of Abnormal Psychology. 1986; 95(3):252-256. [PubMed: 3745647]

Pardini DA, Lochman JE, Powell N. The Development of Callous-Unemotional Traits and Antisocial Behavior in Children: Are There Shared and/or Unique Predictors? Journal of Clinical Child \& Adolescent Psychology. 2007; 36(3):319-333. [PubMed: 17658977]

Pasalich DS, Dadds MR, Hawes DJ, Brennan J. Do callous-unemotional traits moderate the relative importance of parental coercion versus warmth in child conduct problems? An observational study. Journal of Child Psychology and Psychiatry, and Allied Disciplines. 2011; 52(12):13081315.

Patrick CJ. Psychophysiological correlates of aggression and violence: an integrative review. Philosophical Transactions of The Royal Society Biological Science. 2008; 363:254-2555.

Patrick CJ, Hicks BM, Nichol PE, Krueger RF. A bifactor approach to modeling the structure of the psychopathy checklist-revised. Journal of Personality Disorders. 2007; 21:118-41. [PubMed: 17492917]

Patrick C, Hicks B, Krueger R, Lang A. Relations between psychopathy facets and externalizing in a criminal sample. Journal of Personality Disorders. 2005; 19:339-356. [PubMed: 16178678]

Patrick CJ, Bradley MM, Lang PJ. Emotion in the criminal psychopath: startle reflex modulation. Journal of Abnormal Psychology. 1993; 102(1):82-92. [PubMed: 8436703]

Patterson M, Newman J. Reflectivity and learning from aversive events: Toward a psychological mechanism for the syndromes of disinhibition. Psychological Review. 1993; 100(4):716-736. [PubMed: 8255955]

Sadeh N, Spielberg JM, Heller W, Herrington JD, Engels AS, Warren SL, ... Miller GA. Emotion disrupts neural activity during selective attention in psychopathy. Social Cognitive and Affective Neuroscience. 2013; 8(3):235-246. [PubMed: 22210673]

Sadeh N, Verona E. Psychopathic personality traits associated with abnormal selective attention and impaired cognitive control. Neuropsychology. 2008; 22(5):669-680. [PubMed: 18763886]

Sadeh N, Verona E. Visual Complexity Attenuates Emotional Processing in Psychopathy: Implications for Fear-Potentiated Startle Deficits. Cognitive, Affective \& Behavioral Neuroscience. 2012; 12(2):346-360. 
Salekin RT, Worley C, Grimes RD. Treatment of psychopathy: a review and brief introduction to the mental model approach for psychopathy. Behavioral Sciences \& the Law. 2010; 28(2):235-266. [PubMed: 20422648]

Schuckit MA, Tapert S, Matthews SC, Paulus MP, Tolentino NJ, Smith TL, ... Simmons A. fMRI Differences between Subjects with Low and High Responses to Alcohol during a Stop Signal Task. Alcoholism, Clinical and Experimental Research. 2012; 36(1):130-140.

Shipstead Z, Redick TS, Engle RW. Is working memory training effective? Psychological Bulletin. 2012; 138(4):628-654. [PubMed: 22409508]

Simon JR, Rudell AP. Auditory S-R compatibility: the effect of an irrelevant cue on information processing. The Journal of Applied Psychology. 1967; 51(3):300-304. [PubMed: 6045637]

Steffensmeier D, Allan E, Harer M, Streifel C. Age and the Distribution of Crime. The American Journal of Sociology. 1989; 94:803-31.

Sütterlin S, Schroijen M, Constantinou E, Smets E, Bergh OV, den Diest IV. Breath holding duration as a measure of distress tolerance: examining its relation to measures of executive control. Frontiers in Personality Science and Individual Differences. 2013; 4:483.

Skeem JL, Mulvey EP, Appelbaum P, Banks S, Grisso T, Silver E, Robbins PC. Identifying Subtypes of Civil Psychiatric Patients at High Risk for Violence. Criminal Justice and Behavior. 2004; 31(4):392-437.

Stevenson CS, Whitmont S, Bornholt L, Livesey D, Stevenson RJ. A cognitive remediation programme for adults with Attention Deficit Hyperactivity Disorder. Australian and New Zealand Journal of Psychiatry. 2002; 36(5):610-616. [PubMed: 12225443]

Trivedi JK. Cognitive deficits in psychiatric disorders: Current status. Indian Journal of Psychiatry. 2006; 48(1):10-20. [PubMed: 20703409]

Wampold BE. Psychotherapy: The Humanistic (and Effective) Treatment. American Psychologist. 2007; 62:857-873.

Williamson SE, Harpur TJ, Hare RD. Abnormal processing of affective words by psychopaths. Psychophysiology. 1991; 28(3):260-273. [PubMed: 1946892]

Wolf RC, Carpenter RW, Warren CM, Zeier JD, Baskin-Sommers AR, Newman J. Reduced susceptibility to the attentional blink in psychopathic offenders: implications for the attention bottleneck hypothesis. Neuropsychology. 2012; 26(1):102-109. [PubMed: 22023489]

Wykes T, Huddy V, Cellard C, McGurk SR, Czobor P. A Meta-Analysis of Cognitive Remediation for Schizophrenia: Methodology and Effect Sizes. American Journal of Psychiatry. 2011; 168(5):472. [PubMed: 21406461]

Zachary, RA. Shipley Institute of Living Scale: Revised Manual. Los Angeles, CA: WPS, Western Psychological Services; 1986.

Zeier JD, Maxwell JS, Newman J. Attention moderates the processing of inhibitory information in primary psychopathy. Journal of Abnormal Psychology. 2009; 118(3):554-563. [PubMed: 19685952] 
Antisocial
Subtype:

Subtype:

Psychobiological Deficit:

Training

Focus:
Psychopathy<smiles>[Te][Te]</smiles>

Poor Attention

to Context

(measured by behavior and psychophysiology)
Externalizing

$\downarrow$

Poor Affective Cognitive Control (measured by behavior and psychophysiology)

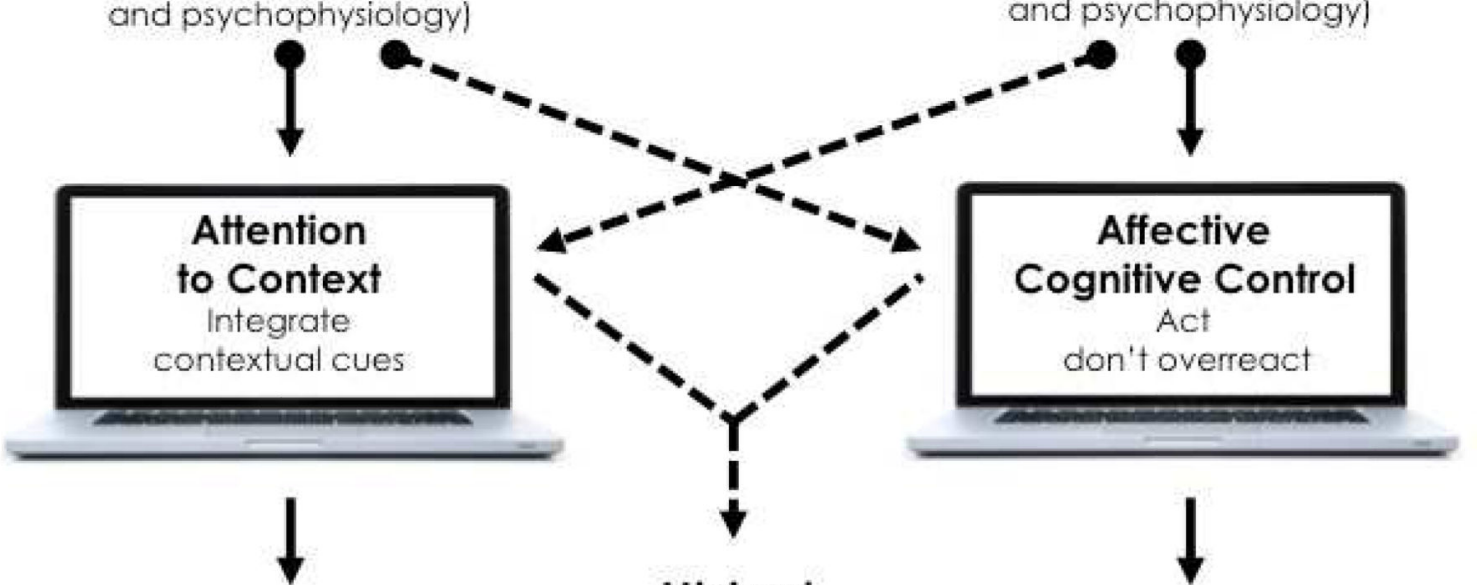

Reduce the

Minimal

Deficit

Change in the Deficit
Reduce the Deficit

Fig. 1. Study Design

Prior to training, all inmates completed a battery of behavioral and psychophysiological assessments that evaluated the cognitive-affective deficits typically associated with these antisocial subtypes. Using a fully crossed $2 \times 2$ design, inmates were then randomly assigned to one of two computerized training programs, where half of the inmates received a cognitive remediation training ( 3 computerized tasks) that matched their specific cognitiveaffective deficit (solid line) and half received a treatment that did not match their deficit (but matched the deficit of the other subtype of antisocial offender; dashed line). At the end of 6 weeks of training, inmates completed a post-training assessment battery that was identical to the one that was administered pre-training. 


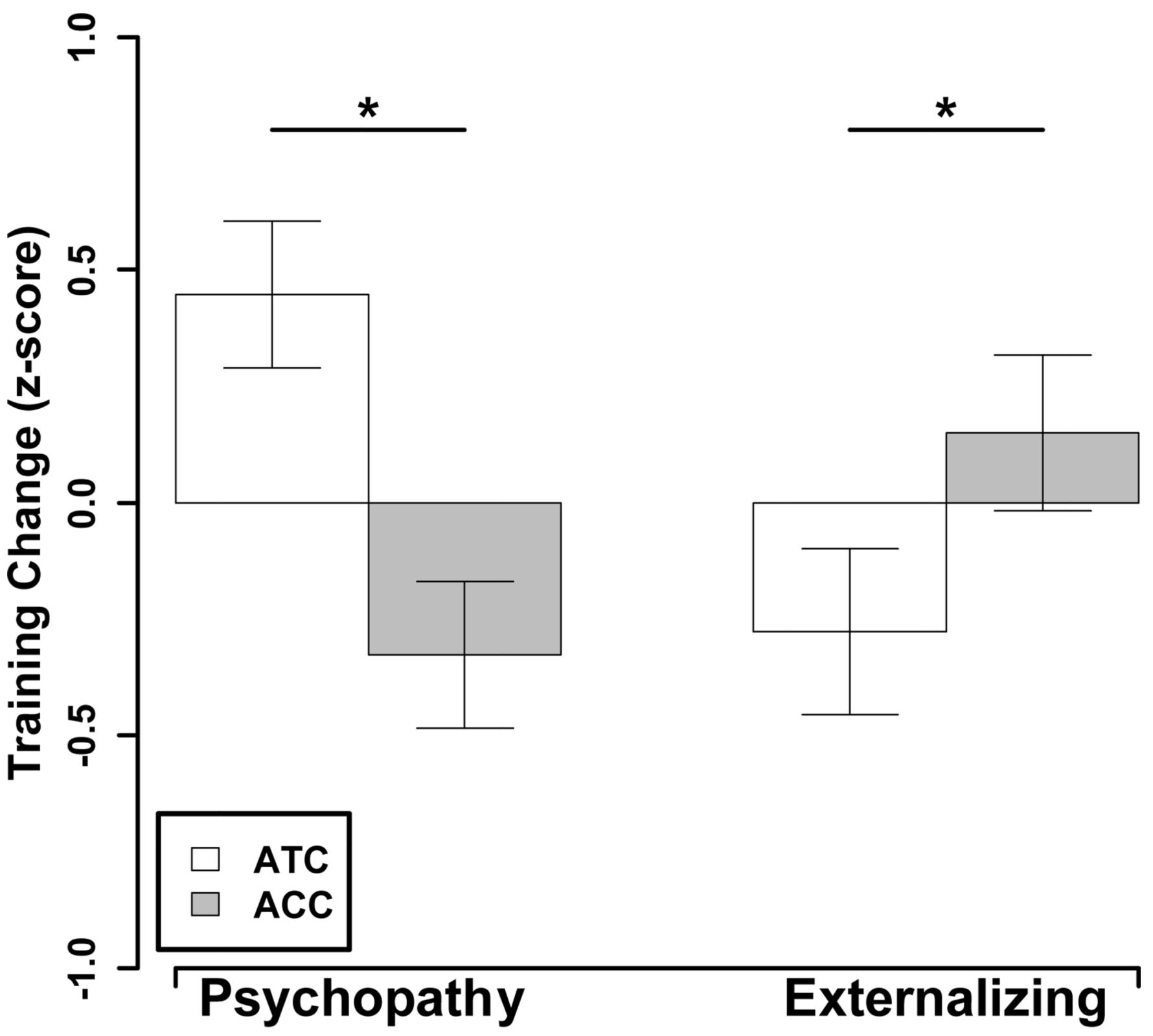

\section{Group}

Fig. 2. Differential Effects of Subtype-Specific Training

Only the targeted antisocial subtype, individuals with psychopathy completing ATC training or individuals with externalizing traits displayed ACC training, displayed significant improvement in performance over the six-week training period. Individuals who received a training that did not match their deficit (i.e., individuals with psychopathy who completed ACC and individuals with externalizing traits who completed ATC) did not show improvement in performance. Asterisks indicate significant differences. ATC $=$ Attention to Context, ACC = Affective Cognitive Control. 


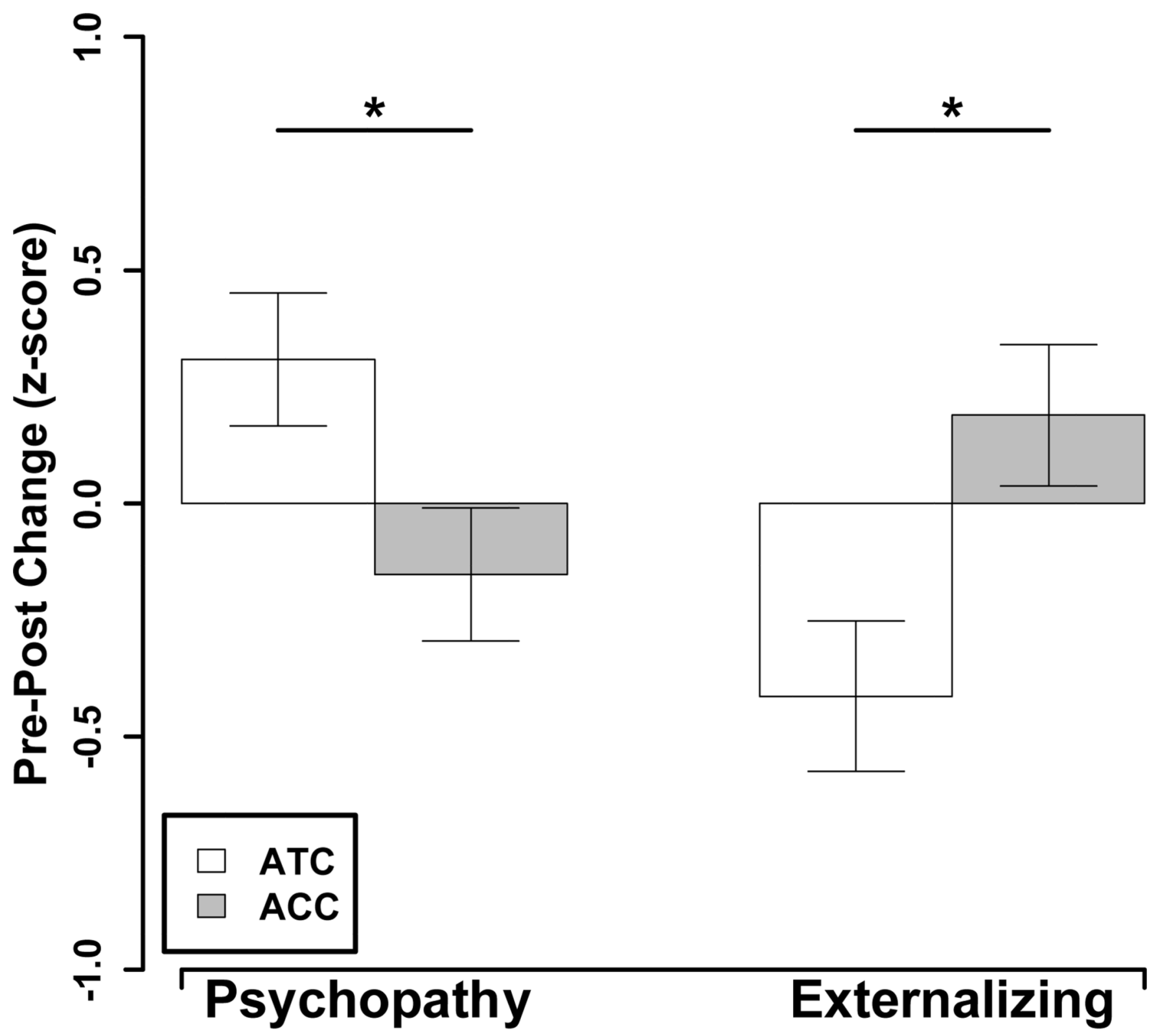

\section{Group}

Fig. 3. Effect of Training on Pre-Post Tasks

Individuals who received the training that matched their cognitive-affective deficit (i.e., individuals with psychopathy who completed ATC and individuals with externalizing traits who completed ACC) displayed significant improvement in performance on tasks that were different than their training. Hence, these individuals showed generalizability of training to other measures. Antisocial individuals who received a training that did not match their deficit (i.e., individuals with psychopathy who completed ACC and individuals with externalizing traits who completed ATC) did not show improvement in cognitive-affective 
functioning. Asterisks indicate significant differences. ATC $=$ Attention to Context, $\mathrm{ACC}=$ Affective Cognitive Control. 